\title{
Monte Carlo generators for the modelling of multijet processes in ATLAS
}

\author{
Evelin Meoni* \\ on behalf of the ATLAS Collaboration \\ Università della Calabria and INFN \\ E-mail: evelin.meonidacern.ch
}

Most of the interesting physics at the LHC involves final states with hadronic jets. We present new Monte Carlo event generator configurations used by the ATLAS experiment to model multijet, boson plus jets and Vector Boson Scattering processes in $p p$ collisions at $13 \mathrm{TeV}$ at the LHC. The different generators are compared to each other and to $13 \mathrm{TeV}$ ATLAS measurements in kinematic distributions sensitive both to the kinematics of hard process and to the effect of shower and nonperturbative effects.

XXVII International Workshop on Deep-Inelastic Scattering and Related Subjects - DIS2019

8-12 April, 2019

Torino, Italy

* Speaker. 


\section{Introduction}

Measurements of multijet final states at the LHC provide precise tests of the perturbative Quantum Chromodynamics (pQCD), the theory describing the strong interactions at short distance. In particular, measurements of inclusive jet cross sections provide interesting inputs for the understanding of basic physics modelling features such as the parton shower or the hadronisation model. Also measurements of the production of a $W / Z$ electroweak vector boson in association with jets ( $W / Z+$ jets) allow to gain a deeper understanding of $\mathrm{pQCD}$ and to test the currently available predictions. Moreover $W / Z+$ jets production is a major background for Higgs-boson measurements, and searches for new physics phenomena, therefore an accurate modelling of the data is needed to constrain the uncertainties. At the LHC is also possible to study the scattering of massive vector bosons (VBS), with two $W / Z$ bosons and two well separated hadronic jets in the final state. Such scattering is a crucial probe of electroweak symmetry breaking. In the Standard Model (SM), the exchange of a Higgs boson ensures unitarity for this process. Many beyond-the-Standard Model (BSM) theories predict enhancements in the VBS rate. VBS is a rare process, but as the LHC experiments accumulate more data, the ability to use these data to constrain BSM theories will depend on having reliable theoretical predictions with well understood systematic uncertainties.

In this contribution, the latest configurations for the Monte Carlo (MC) event generators used by the ATLAS experiment [ [ ] for the modelling of multijet, $W / Z+$ jets and VBS processes at $\sqrt{s}=$ $13 \mathrm{TeV}$ are probed through the comparison with data. For all the studies comprised in this note, jets are built using the anti- $k_{t}$ algorithm [2] with a clustering radius $\mathrm{R}=0.4$.

The contribution is organized as follows: Section $\square$ is dedicated to study of multijet processes, Section [] is focused on $Z+$ jets processes, Section $\mathbb{G}$ describes the studies done on $W^{ \pm} W^{ \pm}+$jets. Finally, Section $\square$ contains the summary and conclusions.

\section{Multi-jet processes}

ATLAS published the measurement of the inclusive jet cross sections with $3.2 \mathrm{fb}^{-1}$ of data in $p p$ collisions at $\sqrt{s}=13 \mathrm{TeV}$ [[]]. Figure $\mathbb{W}$ shows a comparison of the prediction of the latest MC generator configurations with the jet cross sections measured by ATLAS as a function of the inclusive jet momentum $p_{\mathrm{T}}$ for the two extreme bins of jet rapidity: $0<|y|<0.5$ and $1.5<|y|<2.0$. The MC samples investigated are: two samples of dijet production at next-to-leading order (NLO) generated using Herwig 7.1.3, the default angular-ordered parton shower is used for the first sample, while a dipole shower is used for the second one; two samples generated using Sherpa 2.2.5 with the matrix element of the $2 \rightarrow 2$ processes at LO accuracy, the first sample makes use of the dedicated Sherpa AHADIC model for hadronization, based on cluster fragmentation ideas, for the second one the Sherpa interface to the Lund string fragmentation model of Pythia 6.4 is used; a sample generated with Pythia 8.230 with matrix elements of dijet production at LO interfaced to a $p_{\mathrm{T}^{-}}$ ordered parton shower; a sample generated with MadGraph5_aMC@NLO interfaced with Pythia 8.212 (referred to as MG5_aMC@NLO + Py8.212) that has matrix elements computed at LO for up to four final-state partons; a sample generated with Powheg interfaced with Pythia 8.230 (referred to as Powheg + Py8.230) at NLO accuracy. All details on the MC configurations and relative references as well as comparisons for all bins of jet $y$ are provided in [四]. The comparison only 
accounts for the shape of the distributions, as the normalization of each prediction is scaled to the integral of the data. The observation is that the angular-ordered parton showers in Herwig 7.1.3 give the best description of the data for all rapidity ranges, the two Sherpa 2.2.5 samples, generated with different hadronisation models, give also a very good description at forward rapidities, being very similar for both models. It is also important to note that both Pythia 8.230 and MG5_aMC@NLO + Py8.212 significantly differ from the data, predicting lower cross sections for high $p_{\mathrm{T}}$ jets. Powheg + Pythia 8.230 behaves similarly to the Sherpa samples, deviating in the forward rapidity region.
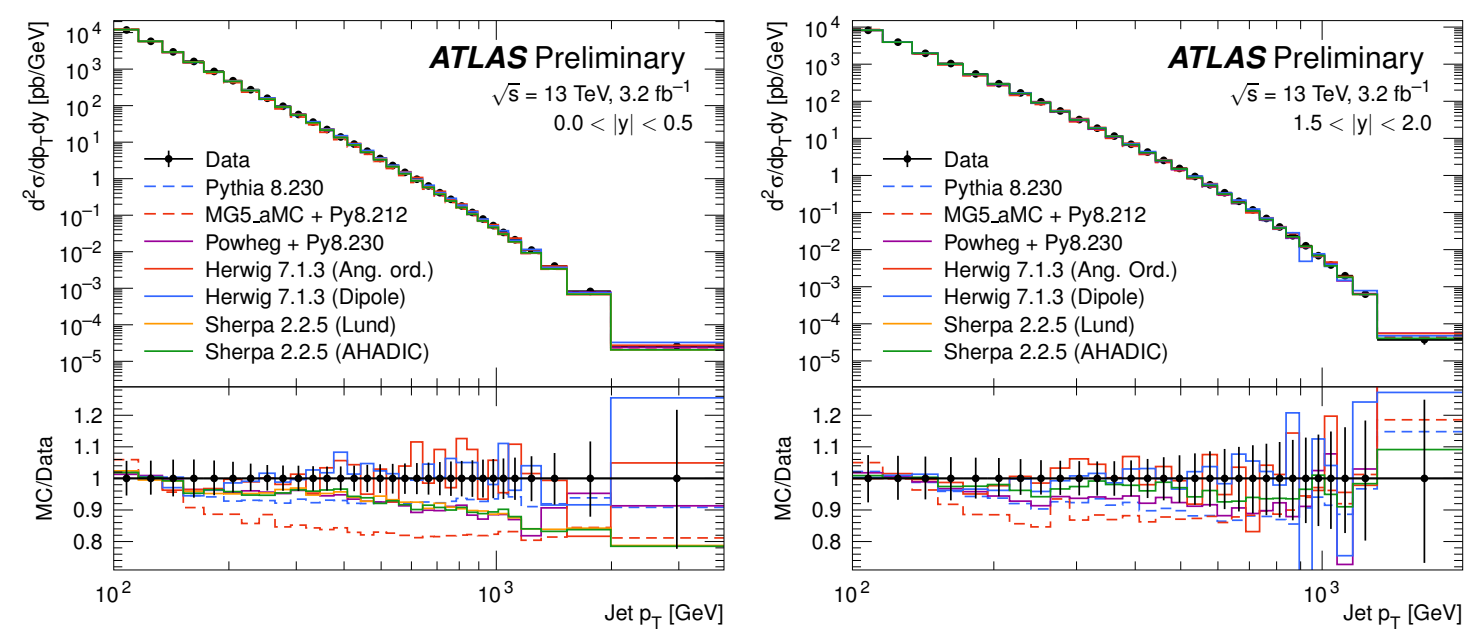

Figure 1: Inclusive jet cross sections as measured by ATLAS [B] as a function of the jet $p_{\mathrm{T}}$ for two rapidity bins. The vertical error bars include both statistical and systematic uncertainties. The lower panel shows the ratio between each MC prediction and the data [四].

\section{Z+jets}

ATLAS published the measurement of the $Z$ boson production in association with jets in a fiducial phase-space as a function of several jet kinamatic observables with $3.2 \mathrm{fb}^{-1}$ of data in $p p$ collisions at $\sqrt{s}=13 \mathrm{TeV}[[]]$. Figure $\square$ shows the comparison of lastest predictions with data for the inclusive and exclusive jet multiplicity distributions. The MC samples investigated are: a sample generated with Sherpa 2.2, it uses the MEPS@NLO setup that is NLO-accurate for up to two extra emissions and LO-accurate for up to four extra emissions; a MadGraph5_aMC@NLO sample with showering and hadronisation performed by Pythia 8 (referred to as MG5 aMC + Py8 FxFx), that has matrix elements for $Z+0,1$ and 2 partons to NLO accuracy; a sample genetared with Powheg MiNLO + Pythia 8 (Py8), that produces NLO accuracy for boson production in association with one jet. All details on the MC configurations and relative references as well as comparisons for the differential cross sections as a function of the scalar jet- $p_{\mathrm{T}}$ sum and the leading jet rapidity are provided in [四]. In Figure $\square$ the measurement uncertainty is indicated by a grey band in the ratio panels, while generator uncertainties have been estimated using Sherpa 2.2 and are indicated by the orange band in the ratio panels. This theory uncertainty band is constructed from the statistical uncertainty of the Sherpa predictions, the parton distribution function (PDF) uncertainties and the 

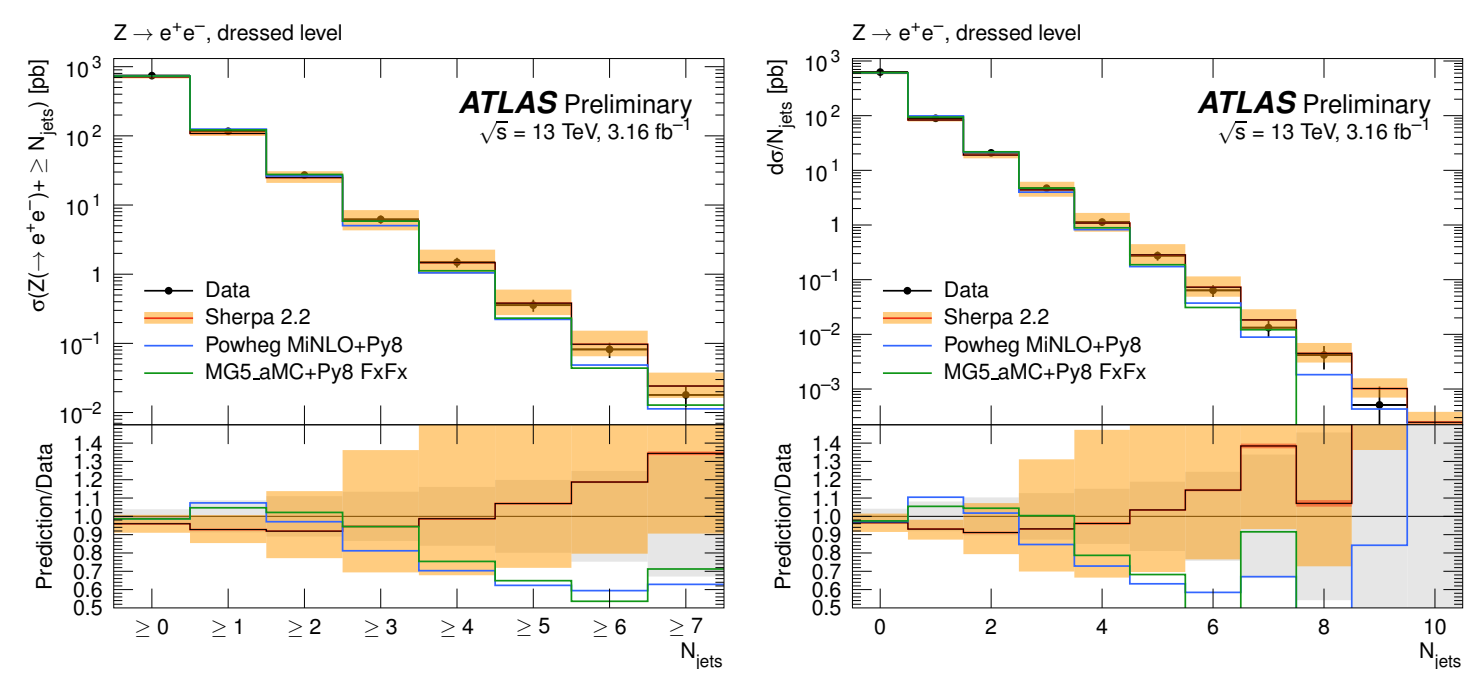

Figure 2: Predictions for the differential cross sections as a function of the inclusive (left) and the exclusive (right) jet multiplicity. Z+jets cross sections as a function of the inclusive (left) and the exclusive (right) jet multiplicity as measured by ATLAS [[]]. The grey band indicates the size of the total measurement uncertainty, while the orange band includes PDF and scale uncertainties estimated with Sherpa 2.2. The size of the statistical uncertainty components are indicated by the size of the error bars [焑].

uncertainties on the renormalisation and factorisation scales. Sherpa 2.2 starts to diverge from the data, indicating too much activity in the parton shower. The MG5_aMC@NLO and Powheg MiNLO + Py8 setups already begin to mismodel the data sooner, making them less attractive for many new-physics searches where a good modelling of high multijet configurations is necessary.

\section{4. $\mathbf{W}^{ \pm} \mathbf{W}^{ \pm}+$jets}

The production of same-sign $W$-bosons in association with two jets $\left(\mathrm{W}^{ \pm} \mathrm{W}^{ \pm} \mathrm{jj}\right)$ is extremely important at the LHC. For this process, with appropriate fiducial cuts on the dijet invariant mass and the rapidity separation between the jets, it is possible to identify a kinematic region where electroweak production, which includes VBS diagrams, dominates over strong production. Observation of $W^{ \pm} W^{ \pm} \mathrm{jj}$ electroweak production has been reported by the CMS Collaboration [8] and by the ATLAS Collaboration [ $[\mathbf{D}]$ using data recorded at a center-of-mass energy of $\sqrt{s}=13 \mathrm{TeV}$. The cross section measured by ATLAS is: $\sigma_{\text {fid }}=2.91_{-0.47}^{+0.51}$ (stat.) \pm 0.27 (syst.) fb

Table $\square$ presents the fiducial cross sections for the several generator configurations in the fiducial region defined by ATLAS Collaboration. The MC samples employed are: different samples generated with MadGraph5_aMC@NLO (referred to as MG5_aMC) both for LO and NLO configurations, showered with Pythia 8 (MG5_aMC + PY8), either with its default $p_{\mathrm{T}}$-ordered shower settings or with a dipole-recoil scheme, or showered with Herwig 7 (MG5_aMC + H7), either with the default angular-ordered model or the dipole-shower one; different POWHEG samples at NLO accuracy showered with options similar to the ones adopted for MG5_aMC; three Sherpa samples at LO accuracy: one sample with matrix element for up to 1 additional parton (SHERPA_LO1), one that includes one additional jet in the matrix element with respect to SHERPA_LO-1 (SHERPA_CKKW) and one with no additional partons (SHERPA_LO-0). SHERPA_LO-1 suf- 


\begin{tabular}{|l|c|}
\hline \multicolumn{2}{|c|}{ Leading-Order Configurations } \\
\hline Sample name & $\sigma[\mathrm{fb}]$ \\
\hline MG5_AMC_LO+PY8 & $3.106 \pm 0.015$ \\
MG5_AMC_LO+PY8,Dipole Recoil & $3.104 \pm 0.015$ \\
MG5_AMC_LO+H7 & $3.016 \pm 0.020$ \\
MG5_AMC_LO+H7,Dipole Shower & $3.022 \pm 0.017$ \\
SHERPA_LO-0 & $2.615 \pm 0.011$ \\
SHERPA_LO-1 & $2.806 \pm 0.046$ \\
\hline
\end{tabular}

\begin{tabular}{|l|c|}
\hline \multicolumn{2}{|c|}{ Leading-Order Multileg Configurations $(\mathbf{0 , 1}$ additional parton) } \\
\hline Sample name & $\sigma[\mathrm{fb}]$ \\
\hline SHERPA_CKKW & $2.048 \pm 0.013$ \\
\hline
\end{tabular}

\begin{tabular}{|l|c|}
\hline \multicolumn{2}{|c|}{ Next-to-Leading-Order Configurations } \\
\hline Sample name & $\sigma[\mathrm{fb}]$ \\
\hline PowHEG + PY8 & $3.122 \pm 0.023_{-0.040}^{+0.050}(\mathrm{scale}) \pm 0.010$ (pdf) \\
PowHEG +PY8,Dipole Recoil & $3.082 \pm 0.023$ \\
PowHEG +H7 & $2.992 \pm 0.026$ \\
PowHEG +H7,Dipole Shower & $3.004 \pm 0.026$ \\
MG5_AMC_NLO+H7, $\Gamma_{\text {resc }}$ & $3.304 \pm 0.033_{-0.040}^{+0.050}($ scale $) \pm 0.010$ (pdf) \\
MG5_AMC_NLO+PY8, $\Gamma_{\text {resc }}$ & $3.345 \pm 0.033$ \\
\hline
\end{tabular}

Table 1: Fiducial cross sections for the process $p p \rightarrow \ell^{ \pm} v \ell^{\prime \pm} v j j$ using the fiducial region defined in [प]. The uncertainties shown correspond to the estimated statistical uncertainty of the Monte Carlo samples. For the POWHEG + PY8 and MG5aMC_NLO + H7 configurations, the scale uncertainty and the PDF uncertainty are also shown [Q].

fers from a non-optimal setting of the color flow setup for the parton shower on top of VBSlike scattering processes, leading to an excess of central emissions from the parton shower [ए]]. SHERPA_CKKW thus corrects for the shape effects of this shower mismodelling, but leads to significantly reduced predicted cross sections due to the large suppression from spuriously large Sudakov factors. While the SHERPA_LO-0 sample is generated to facilitate comparison with other generators. All details on the MC configurations and relative references are provided in [Q]. For the NLO calculations, scale and PDF uncertainties are also provided. These uncertainties are usually small under changes of configuration of the parton shower model and therefore are only provided for one configuration of each NLO generator. Table $\mathbb{W}$ shows that the NLO MG5_aMC cross section is about $10 \%$ higher than LO MG5_aMC. The difference in fiducial cross sections calculated using the two NLO calculations (MG5_aMC and POWHEG) is about 10\%, larger than the uncertainty obtained by combining statistical, scale and PDF uncertainties. One possible source of the observed difference between Powheg and MG5_aMC is the absence of the s-channel diagram in the Powheg configuration. Changes in the choice of shower model for a given hard scattering calculation (e.g. differences between Pythia 8, Herwig 7 and differences between dipole and angular ordered shower) are at most of order 5\%. SHERPA_CKKW predicts a cross section that is 65\% of the MG5_aMC cross section. The source of this discrepancy was discussed above. SHERPA_LO-0 and SHERPA_LO-1 predict rates that are 83\% and 89\% of the MG5_aMC rate respectively, indicating that the non-optimal color flow setup has a smaller effect on the LO con- 
figurations than for the CKKW merged case. POWHEG and MG5_aMC samples are in agreement with data within the quite large data uncertaities, while Sherpa is below of about one $\sigma_{\text {data }}$.

\section{Conclusions}

New event generator configurations for the modelling of jet production at $\sqrt{s}=13 \mathrm{TeV}$ with the ATLAS detector at the LHC have been presented. The predictions of multijet processes are compared to a measurement of the inclusive jet spectrum, a good agreement among the different predictions is found in the region which is dominated by the hard scattering matrix elements. Different variations are produced to estimate uncertainties stemming from the modelling of both the perturbative and non-perturbative parts. Their impact on a few selected distributions is presented and discussed. The baseline Monte Carlo simulation setup used currently by ATLAS to model $Z+$ jets has been described. Discrepancies of predictions with data and between different predictions are highlighted. Finally predictions for electroweak scattering for same-sign $W$ boson pairs have been also presented and the impact of different choices has been discussed. All these studies are of paramount importance for future tunings of MC generators at the LHC.

\section{References}

[1] ATLAS Collaboration, The ATLAS Experiment at the CERN Large Hadron Collider, JINST 3 (2008) S08003

[2] M. Cacciari, G.P. Salam and G. Soyez, The anti- $k_{t}$ jet clustering algorithm, JHEP 073 (2008) 0804, [hep-ph/0802.1189]

[3] ATLAS Collaboration, Measurement of inclusive jet and dijet cross-sections in proton-proton collisions at $\sqrt{s}=13 \mathrm{TeV}$ with the ATLAS detector, JHEP 05 (2018) 195 [hep-ex/1711.02692]

[4] ATLAS Collaboration, Multijet simulation for 13 TeV ATLAS analyses, ATL-PHYS-PUB-2019-017, URL:https://cdsweb.cern.ch/record/2672252

[5] ATLAS Collaboration, Measurements of the production cross section of a $Z$ boson in association with jets in pp collisions at $\sqrt{s}=13$ TeV with the ATLAS detector, Eur. Phys. J. C77 (2017) 361 [hep-ex/1702.05725]

[6] ATLAS Collaboration, ATLAS simulation of boson plus jets processes in Run 2, ATL-PHYS-PUB-2017-006, URL:https://cdsweb.cern.ch/record/2261937

[7] ATLAS Collaboration, Observation of electroweak production of a same-sign $W$ boson pair in association with two jets in pp collisions at $\sqrt{s}=13 \mathrm{TeV}$ with the ATLAS detector, ATLAS-CONF-2018-006, 2018, URL:https://cdsweb.cern.ch/record/2629411

[8] CMS Collaboration, Observation of Electroweak Production of Same-Sign W Boson Pairs in the Two Jet and Two Same-Sign Lepton Final State in ProtonProton Collisions at 13 TeV, Phys. Rev. Lett. 120 (2018) 081801 [hep-ex/1709.05822]

[9] ATLAS Collaboration, Modelling of the vector boson scattering process $p p \rightarrow W^{ \pm} W^{ \pm} j j$ in Monte Carlo generators in ATLAS, ATL-PHYS-PUB-2019-004, URL:https://cdsweb.cern.ch/record/2655303

[10] Stefan Hoche, for the Sherpa collaboration, Status of Sherpa event generator, Multi-Boson Interactions Workshop at the University of Michigan, August 2018 